\title{
Is There A Non-Essential Hospitalization Day In Inpatients With Diabetes Under Medical Insurance? Evidence From An Observational Study In China
}

This article was published in the following Dove Press journal: Diabetes, Metabolic Syndrome and Obesity: Targets and Therapy

\section{Siyu Tao (D) \\ Haomiao $\mathrm{Li}^{1}$ \\ Yueyin $\mathrm{Xie}^{2}$ \\ Jiangyun $\mathrm{Chen}^{3}$ \\ Zhanchun Feng'}

'School of Medicine and Health Management, Tongji Medical College, Huazhong University of Science and Technology, Wuhan, Hubei, People's Republic of China; ${ }^{2}$ School of Automobile, Tongji University, Shanghai, People's Republic of China; ${ }^{3}$ School of Health Service Management, Southern Medical University, Guangzhou, People's Republic of China
Correspondence: Zhanchun Feng School of Medicine and Health Management, Tongji Medical College, Huazhong University of Science and Technology, No. 13 Hangkong Road, Qiaokou District, Wuhan, Hubei 430030 ,

People's Republic of China

Tel +86278369273।

Email zcfeng@hust.edu.cn

Jiangyun Chen

School of Health Service Management, Southern Medical University, No.

1023-1063 Shatainan Road, Baiyun

District, Guangzhou, Guangdong 510515,

People's Republic of China

Tel +86 I85 88220304

Email jy2006/26@|26.com
Purpose: Non-essential hospitalization day of inpatient diabetes threatens health seriously and contributes to great economic burden on individuals and the society. Studying the essential utilization of hospitalization services is conducive to the reduction in the burden of diabetes. The purpose of this study is to identify the existence of non-essential hospitalization days during hospitalization in diabetic patients through exploring the use of health care in different types of insured patients.

Patients and methods: A sample of 6731 admission records from 5929 hospitalized patients was studied. Binary logistic regression was performed to estimate the adjusted effects of health insurance status on readmission. Multiple stepwise linear regression was performed to estimate the adjusted effects of health insurance status on length of stay (LOS), direct medical expenses (DME), out-of-pocket (OOP) expenditures, and percentage of individual payment after reimbursement (PIPAR). Adjusted odds ratios (with 95\% CI) were reported as the results of logistic regression models and linear regression models, respectively.

Results: Adjusted 7-day readmission rate and 30-day readmission rate were not significantly different between urban and rural resident basic medical insurance (URRBMI) and urban employee basic medical insurance (UEBMI). Compared with inpatients under URRBMI, the adjusted LOS and DME were significantly higher for UEBMI inpatients (adjusted OR of 2.6, 95\% CI $=1.9-3.2$, adjusted OR of 1870.85 , 95\% CI=1370.97-2370.73, respectively). Adjusted OOP and PIPAR were significantly lower for UEBMI inpatients (adjusted OR of -970.86 , $95 \% \mathrm{CI}=-1111.63--830.10$, adjusted OR of $-0.19,95 \% \mathrm{CI}=-0.20--0.18$, respectively).

Conclusion: There was a non-essential hospitalization day existing in the treatment of diabetes. Moral hazard has been found in UEBMI which would trigger overtreatment in hospitalization of diabetics, and the lower PIPAR of UEBMI was one of the main causes of moral hazard.

Keywords: diabetes, readmission rate, hospitalization day, medical insurance, moral hazard, overtreatment

\section{Introduction}

According to the World Health Organization (WHO), diabetes, which is the third most serious chronic disease threatening human health after cancer and cardiovascular disease, has become an epidemic in the 21 st century. $^{1}$ In 2017,425 million people worldwide were diagnosed with diabetes, $80 \%$ of whom were in low- and 
middle-income countries, and $25 \%$ of whom were from China. $^{2}$ It cannot be ignored that with population aging, urbanization, industrialization, nutritional changes, high prevalence of obesity and the reduction in physical activity, the incidence of diabetes is growing rapidly. The number of patients with diabetes is estimated to reach 629 million by $2045 .^{3}$ The prevalence of diabetes in China is approximately $11.6 \%,{ }^{4}$ and roughly 90,000 people die from diabetes each year, accounting for $1.39 \%$ of the total deaths. ${ }^{5}$

Both domestic and foreign studies have found that diabetes can place a heavy financial burden on patients, families and the society. ${ }^{6}$ The first global diabetes report from WHO states that the global annual health expenditure on diabetes was more than $\$ 872$ billion in 2016 ; by 2030 , the global economy is expected to lose up to $\$ 1.7$ trillion due to diabetes. ${ }^{7}$ Domestic studies in recent years have shown that diabetes has become the main economic burden of disease in China. The average annual growth rate of total annual medical expenses for diabetes in 2004-2013 was $20.3 \%{ }^{8}$ which exceeded the average annual growth rate of gross domestic product $(15.5 \%),{ }^{9}$ and national health expenditure (14.04\%) during the same period. ${ }^{10}$ This status indicates that the medical care and expense of diabetes are growing sustainably. The proportion of diabetes medical expenses in family health expenditure is $33.99 \%{ }^{11}$

Countries around the world have introduced medical insurance policies, which have stipulated that economic compensation for medical expenses should be given to the insured, in order to protect the basic medical needs of patients and reduce the economic burden of diabetic patients. China has established a basic medical insurance system for urban employees (Urban Employee Basic Medical Insurance, UEBMI) and one for urban and rural residents (Urban and Rural Resident Basic Medical Insurance, URRBMI) according to one's employment situation and the nature of the household registration. ${ }^{12}$ UEBMI covers all in-service employees and retirees of all urban employing units (including enterprises, government organs, public institutions, social organizations and private nonenterprise units); URRBMI covers all urban and rural residents except employees who should be covered by UEBMI. The basic medical insurance system now covers more than 1.35 billion people in China (coverage reached $98.00 \%){ }^{13}$

However, what goes against the intention of medical insurance to reduce the burden on patients is that the occurrence of overtreatment and the growth of unreasonable medical expenses. Several previous investigators have found that type of medical insurance affects the utilization of health services, especially with the development of medical insurance, the increasing reimbursement ratio promoted the release of patient medical service demand and increased the services projects. ${ }^{14-16}$ Domestic and foreign studies have shown that because of the information asymmetry between doctors and patients, the marginal price of medical service reduced by medical insurance and the opportunistic tendency of people, ${ }^{17}$ which will produce moral hazard. ${ }^{18}$ Moral hazard is a situation in which one party gets involved in a risky event knowing that it is protected against the risk and the other party will incur the cost, ${ }^{19}$ which originated in the insurance industry. ${ }^{20}$ Many scholars have studied the welfare losses caused by moral hazard, indicating that moral hazard can induce overtreatment such as non-essential hospitalization days and an increase in medical expenses, ${ }^{21}$ and the economic losses caused by moral hazard may be greater than the gains that obtained by individuals from insurance through moral hazard, ${ }^{22}$ which is not conducive to the development of medical insurance and the reduction of patient burden.

Nonetheless, the current research data on overtreatment and moral hazard in patients with diabetes are rather limited in China, particularly in different basic medical insurance systems. ${ }^{23}$ Simultaneously, since diabetes is a progressive disease that cannot be cured and needs to be treated for lifelong period of time, hospitalized patients with diabetes may have a higher risk of readmission than those without diabetes. ${ }^{24}$ The negative impact of this moral hazard will be even more profound if it is confirmed that moral hazard exists in the use of health care for diabetic patients.

Studying the essential utilization of hospitalization services and moral hazard of diabetics in medical insurance is conducive to the reduction of the burden of diabetes. ${ }^{25,26}$ Therefore, the purpose of this study was to identify the existence of non-essential hospitalization days during hospitalization in diabetic patients through exploring the use of health care in different types of insured patients. The findings of such a study might provide some useful insights for the reconstruction of an improved medical services of diabetics and medical insurance system.

\section{Methods}

\section{Sample Strategy}

The study focused on three capital cities (Wuhan in Hubei Province, Hangzhou in Zhejiang Province, and Chengdu in 
Sichuan Province, located in east, central, and west of China, respectively; and the gross domestic product (GDP) of these cities ranked among the top 10 in the country). The reimbursement and payment levels of the basic medical insurance scheme in the three cities are similar. The study samples are medical records among inpatients with diabetes who were reimbursed by URRBMI and UEBMI in the three selected cities during January 1, 2013 and December 31, 2014. Records were randomly derived with the inpatient's unique identification number from the National Databases for basic medical insurance. A total of 6731 medical records from 5929 inpatients with diabetes were included in the analysis. These data were quality-checked by the Healthcare Security Administration of Wuhan, Hangzhou, and Chengdu. All inpatient information, such as name and address, was excluded before the data were provided to the study team.

The data gathered from the information databases included age, sex, city, sub-diabetes, and diabetes complication condition, type of medical institutions, date of admission, date of discharge, length of stay (LOS), type of insurance, direct medical expenses (DME), out-ofpocket (OOP) expenditures, and percentage of individual payment after insurance reimbursement (PIPAR). And the records of 7-day readmission and 30-day readmission were evaluated according to the admission and discharge time of each hospitalization, because the records of multiple hospital admissions registration numbers could be extracted when screening for the same unique identification number. LOS is a term commonly used to measure the duration of a single episode of hospitalization. ${ }^{27}$ DME refers to the actual cost of hospital stay before medical insurance subsidy, which includes hospital stay, professional fees, medications, tests and ward consumables. The OOP referred to the actual cost paid by patients after medical insurance compensation. PIPAR referred to the proportion of medical expenses that are permitted to be paid under the medical insurance policy, which is borne by the insured individual. This study was performed in accordance with the principles of Helsinki Declaration and was approved by the Ethics Committee of Tongji Medical College, Huazhong University of Science and Technology (IORG No: IORG0003571). The privacy of participants was strictly protected with extracting medical records anonymously.

\section{Data Analysis}

Patient-level covariates and unadjusted outcomes were compared using ANOVA or Kruskal-Wallis test for continuous variables (age, LOS, DME, OOP, PIPAR) and Chi-square test for categorical variables as appropriate: sex, location (middle, east, or west), sub-diabetes (type I, type II, or other types), complication $(0,1$, or $\geq 2)$, type of insurance (URRBMI and UEBMI), and type of medical institutions (basic health care institutions, primary hospitals, secondly hospital, or tertiary hospitals).

Binary logistic regression was performed to estimate the adjusted effects of health insurance status on readmission (7-day readmission and 30-day readmission). Multiple stepwise linear regression was performed to estimate the adjusted effects of health insurance status on LOS, DME, OOP, and PIPAR. Adjusted odds ratios (with 95\% CI) were reported as the results of logistic regression models and linear regression models, respectively.

Categorical variables were presented as percentages and continuous variables as means \pm SD. All statistical analyses were conducted using the Statistical Package for the Social Sciences (SPSS for Windows, version 22.0, IBM Corp., Armonk, NY, USA). Statistical significance was set at $P<0.05$.

\section{Results}

\section{Characteristics Of Sample}

A total of 6731 admission records were extracted, with a mean (SD) age of 55.0 (13.4) years, and 2513 (37.3\%) were male. More than $98 \%$ of visits were inpatients with type II diabetes. A total of 1199 visits $(17.8 \%)$ had at least one complication, and $170(2.5 \%)$ had two or more complications. A total of 1132 visits (16.8\%) were insured by URRBMI, and percentage of individual payment after URRBMI reimbursement was $46.4 \%$, which was much higher than UEMBI (28.9\%). Two-thirds (66.1\%) visited tertiary hospitals, and only one-twentieth $(5.2 \%)$ visited basic medical institutions (community health care centers, township health centers, or village clinics). The frequencies of patient characteristics stratified on the basis of health insurance status are listed in Table 1.

Sub-diabetes and diabetes complication conditions were not significantly different between URRBMI and UEBMI inpatients. Inpatients under URRBMI group were generally elderly than those from the UEBMI group. Most (67.9\%) URRBMI inpatients were female, which was twice to the UEBMI inpatients (31.1\%). The 
Table I Characteristics Of Inpatients With Diabetes By Basic Health Insurance Status

\begin{tabular}{|c|c|c|c|c|}
\hline \multirow[t]{2}{*}{ Characteristics } & \multirow[b]{2}{*}{ All Patients $(n=6731)$} & \multicolumn{2}{|l|}{ Insurance } & \multirow[t]{2}{*}{$P$ Value } \\
\hline & & URRBMI $(n=|| 32)$ & UEBMI $(n=5599)$ & \\
\hline Age, mean (SD), y & $55.0(13.4)$ & $61.5(14.1)$ & $53.7(12.9)$ & $<0.001$ \\
\hline Female sex & $2513(37.3)$ & $769(67.9)$ & $1744(31.1)$ & $<0.001$ \\
\hline $\begin{array}{l}\text { Location } \\
\text { Middle } \\
\text { East } \\
\text { West }\end{array}$ & $\begin{array}{l}4804(71.4) \\
589(8.8) \\
1338(19.9)\end{array}$ & $\begin{array}{l}796(70.3) \\
43(3.8) \\
293(25.9)\end{array}$ & $\begin{array}{l}4008(71.6) \\
546(9.8) \\
1045(18.7)\end{array}$ & $<0.001$ \\
\hline $\begin{array}{l}\text { Sub-diabetes } \\
\text { Type I DM } \\
\text { Type II DM } \\
\text { Other types of DM (GDM) }\end{array}$ & $\begin{array}{l}110(1.6) \\
6607(98.2) \\
14(0.2)\end{array}$ & $\begin{array}{l}25(2.2) \\
\mathrm{I} 106(97.7) \\
\mathrm{I}(0.1)\end{array}$ & $\begin{array}{l}85(1.5) \\
550 \mid(98.2) \\
13(0.2)\end{array}$ & 0.156 \\
\hline $\begin{array}{l}\text { Complication condition, No. } \\
\qquad \begin{array}{l}0 \\
1 \\
\geq 2\end{array}\end{array}$ & $\begin{array}{l}5532(82.2) \\
1029(15.3) \\
170(2.5)\end{array}$ & $\begin{array}{l}928(82.0) \\
170(15.0) \\
34(3.0)\end{array}$ & $\begin{array}{l}4604(82.2) \\
859(15.3) \\
136(2.4)\end{array}$ & 0.521 \\
\hline $\begin{array}{l}\text { Type of medical institutions } \\
\text { Basic health care institutions } \\
\text { Primary hospitals } \\
\text { Secondly hospital } \\
\text { Tertiary hospitals }\end{array}$ & $\begin{array}{l}348(5.2) \\
401(6.0) \\
1536(22.8) \\
4446(66.1)\end{array}$ & $\begin{array}{l}90(8.0) \\
63(5.6) \\
267(23.6) \\
712(62.9)\end{array}$ & $\begin{array}{l}258(4.6) \\
338(6.0) \\
1269(22.7) \\
3734(66.7)\end{array}$ & $<0.001$ \\
\hline
\end{tabular}

Abbreviations: DM, diabetes m mellitus; GDM, gestational diabetes mellitus; URRBMI, urban and rural resident basic medical insurance; UEBMI, urban employee basic medical insurance.

percentage of tertiary hospital admission was highest for URRBMI (62.9\%) and UEBMI (66.7\%) inpatients, and lowest admission for URRBMI and UEBMI inpatients were primary hospitals $(5.6 \%)$ and basic health care institutions $(4.6 \%)$, respectively.

\section{Unadjusted Outcomes For The Effect Of Basic Health Insurance Status}

Table 2 presents the unadjusted outcomes of the basic health insurance groups. The 7-day readmission rate and 30-day readmission rate were not significantly between URRBMI $(0.8 \%$ and $3.2 \%$, respectively) and UEBMI $(0.9 \%$ and $3.9 \%$, respectively) inpatients ( $\mathrm{p}>0.05)$.

Generally speaking, inpatients in the UEBMI group resulted in longer LOS than those from the URRBMI group $(p<0.001)$. The mean LOS for URRBMI and UEBMI inpatients was $10.8 \pm 7.4$ days and $12.5 \pm 10.3$ days, respectively. The mean DME for URRBMI and UEBMI inpatients was $6001.4 \pm 7613.0 \mathrm{CNY}(\$ 894.60 \pm 1134.83)$ and $7722.6 \pm 8549.1$ CNY (\$1151.17 \pm 1274.37$)$, respectively. The mean OOP and PIPAR in URRBMI group were higher than UEBMI, with OOP of $2916.9 \pm 2559.6 \mathrm{CNY}(\$ 434.81 \pm 381.55)$ and 1937.7
Table 2 Unadjusted Outcomes For Effect Of Insurance Status Among Inpatients With Diabetes

\begin{tabular}{|l|l|l|l|}
\hline Outcomes & URRBMI & UEBMI & $\begin{array}{l}\boldsymbol{P} \\
\text { Value }\end{array}$ \\
\hline 7-day readmission (\%) & 0.8 & 0.9 & 0.735 \\
30-day readmission (\%) & 3.2 & 3.9 & 0.304 \\
LOS (days) & $10.8 \pm 7.4$ & $12.5 \pm 10.3$ & $<0.001$ \\
DME (CNY) & $6001.4 \pm 7613.0$ & $7722.6 \pm 8549.1$ & $<0.001$ \\
OOP (CNY) & $2916.9 \pm 2559.6$ & $1937.7 \pm 2185.8$ & $<0.001$ \\
PIPAR (\%) & 46.4 & 28.9 & $<0.001$ \\
\hline
\end{tabular}

Note: ICNY=0.15 Dollar.

Abbreviations: LOS, length of stay; DME, direct medical expenses; OOP, out-ofpocket expenditures; PIPAR, percentage of individual payment after insurance reimbursement; URRBMI, urban and rural resident basic medical insurance; UEBMI, urban employee basic medical insurance.

$\pm 2185.8 \mathrm{CNY}(\$ 288.84 \pm 325.83)$, PIPAR of $46.4 \%$ and $28.9 \%$, respectively $(\mathrm{p}<0.001)$.

\section{Adjusted Outcomes For The Effect Of Basic Health Insurance Status}

Table 3 shows the outcomes for the effect of basic health insurance status among inpatients with diabetes after 
Table 3 Adjusted Outcomes For Effect Of Insurance Status Among Inpatients With Diabetes

\begin{tabular}{|l|l|l|}
\hline Outcomes & URRBMI & UEBMI \\
\hline 7-day readmission & 1.00 & $0.72(0.47$ to $1.1 \mathrm{I})$ \\
30-day readmission & 1.00 & $1.01(0.76$ to 1.32$)$ \\
LOS & 1.00 & $2.6(1.9$ to 3.2$)$ \\
DME & 1.00 & $1870.85(1370.97$ to 2370.73$)$ \\
OOP & 1.00 & $-970.86(-1111.63$ to -830.10$)$ \\
PIPAR & 1.00 & $-0.19(-0.20$ to -0.18$)$ \\
\hline
\end{tabular}

Notes: 7-day readmission, 30-day readmission reported as adjusted OR $(95 \% \mathrm{Cl})$ Length of stay, direct medical expenses, out-of-pocket charges, and percentage of individual payment after reimbursement reported as incidence rate ratio $(95 \% \mathrm{Cl})$. Reference group: URRBMI. Outcomes adjusted for patient's age, gender, location, health insurance status, sub-diabetes, complication condition, medical institutions type. $\mathrm{ICNY}=0.15$ Dollar.

adjustment for age, gender, location, health insurance status, sub-diabetes, complication condition, and type of medical institutions.

Adjusted 7-day readmission rate and 30-day readmission rate were not significantly between URRBMI and UEBMI. Compare to URRBMI inpatients, adjusted LOS and DME were significantly higher for UEBMI inpatients (adjusted OR of 2.6, 95\% CI=1.9-3.2, adjusted OR of $1870.85,95 \%$ $\mathrm{CI}=1370.97-2370.73$, respectively). Adjusted OOP and PIPAR were significantly lower for UEBMI inpatients (adjusted OR of $-970.86,95 \% \mathrm{CI}=-1111.63-830.10$, adjusted $\mathrm{OR}$ of $-0.19,95 \% \mathrm{CI}=-0.20-0.18$, respectively).

\section{Bivariate Correlations For Outcomes By Insurance Status}

For URRBMI group, bivariate correlations tests showed a negative correlation between LOS and PIPAR, with $\mathrm{r}=-0.182(\mathrm{p}<0.001)$, but a positive correlation between LOS, DME, OOP, and PIPAR $(\mathrm{r}=0.199-0.907$, $\mathrm{p}<0.001)$ (Table 4).

For UEBMI group, the results showed that there was a negative correlation between DME and PIPAR, with $\mathrm{r}$ of $-0.259(\mathrm{p}<0.001)$. Besides, there might be a positive correlation between LOS, DME, OOP, and PIPAR $(\mathrm{r}=0.313-0.753, \mathrm{p}<0.001)$ (Table 4).

\section{Discussion}

In this observational study, we have identified the existence of non-essential hospitalization days during hospitalization for diabetic patients based on the readmission rate, length of stay, and insurance-related indicators. The results suggested that there was no difference in the 7-day readmission rate in URRBMI and UEBMI insured patients $(0.8 \%$ and $0.9 \%$,
Table 4 Bivariate Correlations For Outcomes Among Inpatients With Diabetes By Insurance Status

\begin{tabular}{|c|l|l|l|l|}
\hline Category & LOS & DME & OOP & PIPAR \\
\hline $\begin{array}{c}\text { URRBMI } \\
\text { LOS }\end{array}$ & 1.000 & & & \\
DME & $0.308^{* *}$ & 1.000 & & \\
OOP & $0.211^{* *}$ & $0.907 * *$ & 1.000 & \\
PIPAR & $-0.182^{* *}$ & $0.199 * *$ & $0.503^{* *}$ & 1.000 \\
\hline UEBMI & & & & \\
LOS & 1.000 & & & \\
DME & $0.594^{* *}$ & 1.000 & & \\
OOP & $0.360^{* *}$ & $0.753^{* *}$ & 1.000 & \\
PIPAR & $0.324^{* *}$ & $-0.259 * *$ & $0.313^{* *}$ & 1.000 \\
\hline
\end{tabular}

Note: **Correlation is significant at the 0.01 level (2-tailed).

Abbreviations: LOS, length of stay; DME, direct medical expenses; OOP, out-ofpocket expenditures; PIPAR, percentage of individual payment after insurance reimbursement; URRBMI, urban and rural resident basic medical insurance; UEBMI, urban employee basic medical insurance.

respectively) after adjustment for initial differences in individual characteristics, location, health insurance status, subdiabetes, complication condition, and medical institutions type. The finding was similar for the 30-day readmission rate in URRBMI and UEBMI insured patients $(3.2 \%$ and $3.9 \%$, respectively). As we knew, the readmission rate is a sensitive indicator of the quality of inpatient medical services in hospital management theory and practice. ${ }^{28}$ These results indicated that there was no significant difference in the quality of hospitalized medical services received by both URRBMI and UEBMI diabetic inpatients. Meanwhile, the results showed that the LOS of UEBMI insured inpatients was an average of 2.6 days more than that of URRBMI insured inpatients, after adjustment for age, gender, location, health insurance status, sub-diabetes, complication condition, type of medical institutions. So, why is the LOS of hospitalized patients with UEBMI insurance longer, even if they received a similar quality of hospitalized medical services as URRBMI hospitalized patients? There might be non-essential hospitalization days in the hospitalization of diabetic inpatients under UEBMI.

The results of bivariate correlations tests showed that, in UEBMI insured group, there was a negative correlation between DME and PIPAR, and a positive correlation between DME and OOP. Compared to URRBMI insured inpatients, there were $1870.85 \mathrm{CNY}(\$ 278.88)$ more in DME, 970.86 CNY (\$ 144.72) less in OOP, and 19\% less in PIPAR of UEBMI insured inpatients on average. On one hand, some lower DME inpatients may not have reached the deductible line of UEBMI medical insurance 
and therefore, have to pay in OOP all by themselves, which could explain that lower DME inpatients have a relatively higher PIPAR. On the other hand, as to the that higher DME inpatients have a relatively lower PIPAR, more than $70 \%$ of DME was paid according to the policy of UEBMI, there could be induced use of inpatient service even when they have the same higher OOP. Therefore, it can be considered that there is moral hazard in UEBMI, which would trigger overtreatment such as an unduly long rest for a slight illness and outpatient conversion to hospitalization.

The payment mechanism of medical insurance determines the health protection function of the medical insurance system. ${ }^{29}$ The reimbursement policies of the UEBMI, by contrast, are generally superior to URRBMI in China, and the PIPAR nationwide for URRBMI and UEBMI in 2015 were $39.8 \%$ and $27.2 \%$, respectively. ${ }^{30}$ In this study, the actual medical expense of URRBMI and UEBMI $(46.4 \%, 28.9 \%$, respectively) was higher than national level, especially for URRBMI. In addition, the results showed that the PIPAR of UEBMI was much lower than that of URRBMI (19\% on average). In this case, the motivation of inpatients insured by UEBMI to prevent risks reduced and incentives for overuse of medical services increased, so the opportunistic behavior is more likely to occur, resulting in non-essential hospitalization days. Therefore, lower PIPAR of UEBMI may be one of the main reasons for moral hazard.

Differences in fundraising for medical insurance bring differences in compensation. ${ }^{31}$ Different from the fundraising mode of "individual contribution and government subsidy" of URRBMI, the expenses of UEBMI are paid jointly by employers and individual employees. ${ }^{32}$ The fundraising level of UEBMI is higher than that of URRBMI, so employees generally enjoy a higher level of medical security. ${ }^{33} \mathrm{At}$ present, the actual burden ratio of employers and individuals is 3.5:1 in UEBMI, which is much higher than the $50 \%$ burden of employers and employees in most countries. $^{34,35}$ The actual burden of the individual is too low, which will weaken the essence of insurance system and cause the moral hazard of medical insurance. ${ }^{36}$ The individual co-payment rate should be controlled to an appropriate extent, which can achieve the purpose of sharing the risk of disease and reducing the occurrence of moral hazard. In addition, referring to the medical insurance fundraising policy implemented by some countries in the world for retirees, it may help to reduce the moral hazard of retirees using UEBMI. ${ }^{37}$
Meanwhile, previous researches have showed that hospitalization days are positively related to medical expense. ${ }^{38,39}$ Bivariate correlations test of this study also found a positive correlation between LOS and DME in both URRBMI and UEBMI groups. Non-essential hospitalization days have been shown to bring economic burden and health risks to patients, while increasing the risk of basic health insurance funds. ${ }^{40}$ From 2009 to 2013 , the average annual growth rate of medical expenses in China was $15.91 \%$. The average annual growth rate of hospitalization expenses of URRBMI and UEBMI was $25.65 \%$, which was higher than the average annual growth rate of GDP in China during the same period $(10.12 \%){ }^{41}$ At present, the average annual growth rate of fund expenditure of URRBMI and UEBMI is higher than that of fundraising, which indicates that the expenditure pressure of basic health insurance in China is relatively high. Through literature research, it is found that non-essential hospitalization days are not only related to moral hazard, but also related to medical staff's irregular medical behavior and unreasonable medical insurance payment. ${ }^{36,42}$ In order to prevent moral hazard, policymakers should strengthen information construction, dynamic supervision, and the reform of medical insurance payment methods. In addition, informationized blood glucose management and case management mode could be explored in the treatment of diabetes, which have been used abroad maturely and have achieved remarkable results in improving the quality of medical treatment and nursing, reducing hospitalization days and the medical expenses. $^{43-45}$

\section{Limitations}

This study had several limitations. Firstly, the research data were extracted from the official medical insurance management system, so this study was based on the available variables which may not be sufficient. Second, the characteristics and behavioral preferences of doctors may influence the results of the study, but we could not assess the impact of doctors on the utilization of hospitalization service and medical insurance through available data given. Further analyses should be conducted on more variables, especially at the doctor level.

\section{Conclusion}

In conclusion, there is a non-essential hospitalization day existing in the treatment of diabetes. This study indicates that there is moral hazard in UEBMI which would trigger overtreatment in hospitalization of diabetics, and the lower PIPAR of UEBMI is one of the main causes of moral 
hazard. On the basis of the survey, this study recommends an appropriate increase in the individual co-payment rate, the medical insurance fundraising policy for retirees and the reform of medical insurance payment to prevent moral hazard in China. This study also suggests the development of treatment process supervision and diabetes management model to reduce unnecessary hospitalization days.

\section{Abbreviations}

WHO, World Health Organization; URRBMI, urban and rural resident basic medical insurance; UEBMI, urban employee basic medical insurance; GDP, gross domestic product; LOS, length of stay; DME, direct medical expenses; OOP, out of pocket; PIPAR, percentage of individual payment after insurance reimbursement.

\section{Ethics And Consent}

The study was approved by the Ethics Committee of Tongji Medical College, Huazhong University of Science and Technology (IORG No: IORG0003571). This study has been performed in accordance with the ethical standards as laid down in the 1964 Declaration of Helsinki and its later amendments. The data used in this study were obtained from the management organization of medical insurance with its permission, and information related to inpatients' privacy was not extracted.

\section{Availability Of Data And Materials Statement}

According to the statistic law of People's Republic of China and the confidentiality agreement between research team and the Healthcare Security Administration, the research team must keep the security of the initial data. The datasets supporting the conclusions of this article may be available upon any reasonable request from the corresponding author.

\section{Acknowledgments}

The authors appreciate all participants of this study. The authors thank the Healthcare Security Administration of Wuhan city, Hangzhou city, and Chengdu city, for their willingness to provide us with the data.

\section{Author Contributions}

All authors contributed to conception and design, data acquisition and analysis, drafting or revising the article, gave final approval of the version to be published, and agree to be accountable for all aspects of the work.

\section{Disclosure}

The authors report no conflicts of interest in this work.

\section{References}

1. Society CMAD. Guidelines for the prevention and treatment of type 2 diabetes in China (2013 edition). Chin J Diabetes. 2014;7(6):447-498.

2. Anonymous. Q\&A: key points for IDF Diabetes Atlas 2017. Diabetes Res Clin Pr. 2018;135:235-236.

3. Whiting DR, Guariguata L, Weil C, Shaw J. IDF diabetes atlas: global estimates of the prevalence of diabetes for 2011 and 2030 . Diabetes Res Clin Pr. 2011;94(3):311-321.

4. Shaw JE, Sicree RA, Zimmet PZ. Global estimates of the prevalence of diabetes for 2010 and 2030. Diabetes Res Clin Pract. 2010;87(1):4-14.

5. Xu Y, Wang L, He J, et al. Prevalence and control of diabetes in Chinese adults. JAMA. 2013;310(9):948-959.

6. Dall TM, Yang W, Halder P, et al. The economic burden of elevated blood glucose levels in 2012: diagnosed and undiagnosed diabetes, gestational diabetes mellitus, and prediabetes. Diabetes Care. 2014;37(12):3172-3179.

7. Jing Z, Chu J, Imam SZ, et al. Catastrophic health expenditure among type 2 diabetes mellitus patients: a province-wide study in Shandong, China. J Diabetes Investig. 2019;10(2):283-289.

8. Nan X, Kejun L, Xuefei G, et al. The research on total medical expenses of patients with diabetes. Chin Health Econ. 2016;10:65-68.

9. China NBOS. China Statistical Yearbook-2018. Beijing: China Statistics Press; 2018.

10. Daren Z, Tao Z, Bin X, Zhihui L, Sichang H, Jiaoyue L. Analysis and prediction research of total expenditure on health in China between 2004 and 2014. Chin J Med Manage Sci. 2016;4:36-40.

11. Yuhui Z, Quan W, Peipei C, Feng G, Xiufeng W. The medical costs and financing burden of diabetes mellitusin China. Chin Health Econ. 2017;4:17-19.

12. Su D, Chen Y, Gao H, et al. Effect of integrated urban and rural residents medical insurance on the utilisation of medical services by residents in China: a propensity score matching with difference-indifferences regression approach. BMJ Open. 2019;9(2):e0264082.

13. Yinglun S. China Sees Progresses of Community-Level Healthcare. Vol. 2019: Xinhua; 2019.

14. Zhang Y, Zhang L, Li H, Chen Y. Determinants of inappropriate admissions in county hospitals in Rural China: a cross-sectional study. Int J Env Res Pub He. 2018;15(6):1050.

15. Chang J, Chen Y, Gao H, et al. Effect of inappropriate admissions on hospitalization performance in county hospitals: a cross-sectional study in rural China. Cost Effect Resour A. 2019;17:8.

16. Blecker S, Herrin J, Li L, Yu H, Grady JN, Horwitz LI. Trends in hospital readmission of medicare-covered patients with heart failure. J Am Coll Cardiol. 2019;73(9):1004-1012.

17. Boonen LHHM, Laske-Aldershof T, Schut FT. Switching health insurers: the role of price, quality and consumer information search. Eur J Health Econ. 2016;17(3):339-353.

18. Arrow KJ. Uncertainty and the welfare economics of medical care. $J$ Health Polit Polic. 2001;26(5):851-883.

19. Ericson R, Barry D, Doyle A. The moral hazards of neoliberalism: lessons from the private insurance industry. Econ Soc. 2000;29 (4):532-558.

20. Rowell D, Connelly LBA. History of the term "Moral Hazard". $J$ Risk Insur. 2012;79(4):1051-1075.

21. Dranove D. Demand inducement and the physician/patient relationship. Econ Inq. 1988;26(2):281-298. 
22. Feldman R, Sloan F. Competition among physicians, revisited. $J$ Health Polit Policy Law. 1988;13(2):239-261.

23. Jun W, Lidan W, Qicheng J, Weizhen D. Direct medical costs of diabetic patients in China: a systematic review. Mod Preventive Med. 2018;45(3):462-490.

24. Rubin DJ. Hospital readmission of patients with diabetes (vol 15, 17, 2015). Curr Diabetes Rep. 2018;18:214.

25. Chandoevwit W, Phatchana P. Inpatient care expenditure of the elderly with chronic diseases who use public health insurance: disparity in their last year of life. Soc Sci Med. 2018;207:64-70.

26. Nguyen NH, Khera R, Ohno-Machado L, Sandborn WJ, Singh S. Annual burden and costs of hospitalization for high-need, high-cost patients with chronic gastrointestinal and liver diseases. Clin Gastroenterol Hepatol. 2018;16(8):1284-1292.

27. Smith RJ, Santamaria JD, Faraone EE, Holmes JA, Reid DA, Tobin AE. The duration of hospitalization before review by the rapid response team: a retrospective cohort study. J Crit Care. 2015;30(4):692-697.

28. Krumholz HM, Lin Z, Keenan PS, et al. Relationship between hospital readmission and mortality rates for patients hospitalized with acute myocardial infarction, heart failure, or pneumonia. JAMA. 2013;309(6):587-593.

29. Getmatets O, Matviichuk A, Korobtsova D, Kotlyar A, Telestakova A. [Financial and legal guaranteeing of medical insurance in Ukraine]. Georgian Med News. 2018;284:149-154.

30. Srcurity TSIA. Annual Report on China's Social Insurance Development in 2015. Beijing: China Labor Social Security Publishing House; 2016.

31. Zhu K, Zhang L, Yuan S, Zhang X, Zhang Z. Health financing and integration of urban and rural residents' basic medical insurance systems in China. Int J Equity Health. 2017;16(1):194.

32. Pan Y, Chen S, Chen M, et al. Disparity in reimbursement for tuberculosis care among different health insurance schemes: evidence from three counties in central China. Infect Dis Poverty. 2016;5(1):7.

33. Meng Y, Han J, Qin S. The impact of health insurance policy on the health of the senior floating population-evidence from China. Int $J$ Env Res Pub He. 2018;15(10):215910.
34. Colla CH, Dow WH, Dube A. San Francisco's 'Pay Or Play' employer mandate expanded private coverage by local firms and a public care program. Health Affair. 2013;32(1):69-77.

35. Liu Y, Jin GZ. Employer contribution and premium growth in health insurance. J Health Econ. 2015;39:228-247.

36. Einav L, Finkelstein A, Ryan SP, Schrimpf P, Cullen MR. Selection on moral hazard in health insurance. Am Econ Rev. 2013;103(1):178-219.

37. Buetler M. Social security programs and retirement around the world: fiscal implications of reform. J Pension Econ Finan. 2009;8(3):400-401.

38. Fanikos J, Rao A, Seger AC, Carter D, Piazza G, Goldhaber SZ. Hospital costs of acute pulmonary embolism. Am J Med. 2013;126 (2):127-132.

39. Xie F, Jiang X, Yuan F, Chen X, Yuan Z, Lu Y. Impact of the new cooperative medical scheme on the rural residents' hospitalization medical expenses: a five-year survey study for the Jiangxi Province in China. Int J Env Res Pub He. 2018;15(7):13687.

40. Mao W, Zhang L, Chen W. Progress and policy implication of the Insurance Programs for Catastrophic Diseases in China. Int $J$ Health Plan M. 2017;32(3SI):299-306.

41. Li Q, Chen F, Yang M, et al. The effect of China's national essential medicine policy on health expenses: evidence from a national study. Inquiry J Health Car. 2018;55:0046958018787057.

42. Atinga RA, Mensah SA, Asenso-Boadi F, Adjei FA. Migrating from user fees to social health insurance: exploring the prospects and challenges for hospital management. BMC Health Serv Res. 2012;12(1):174.

43. Hsu C, Tai T. Long-term glycemic control by a diabetes case-management program and the challenges of diabetes care in Taiwan. Diabetes Res Clin Pr. 2014;106:S328-S332.

44. Shin SA, Kim H, Lee K, Lin V, Liu G, Shin E. Effects of diabetic case management on knowledge, self-management abilities, health behaviors, and health service utilization for diabetes in Korea. Yonsei Med J. 2015;56(1):244-252.

45. Huang X, Liu J, Lyu F, et al. Effect of "hospital informationized blood glucose management" on perioperative diabetic patients. Chin J Endocrinol Metab. 2018;34:768-772.

\section{Publish your work in this journal}

Diabetes, Metabolic Syndrome and Obesity: Targets and Therapy is an international, peer-reviewed open-access journal committed to the rapid publication of the latest laboratory and clinical findings in the fields of diabetes, metabolic syndrome and obesity research. Original research, review, case reports, hypothesis formation, expert opinion and commentaries are all considered for publication. The manuscript management system is completely online and includes a very quick and fair peer-review system, which is all easy to use. Visit http://www.dovepress.com/testimonials.php to read real quotes from published authors. 